\title{
Leiomiosarcoma de duodeno: reporte de caso
}

\section{A Case of Duodenal Leiomyosarcoma}

Rafael Parra-Medina, MD, ${ }^{1}$ Paula Moreno L., ${ }^{2}$ Julián Jiménez M., ${ }^{2}$ Marcela Sánchez R., MD, ${ }^{3}$ Edgardo Yaspe C., MD, ${ }^{4}$ Patricia López C., MD. ${ }^{5}$

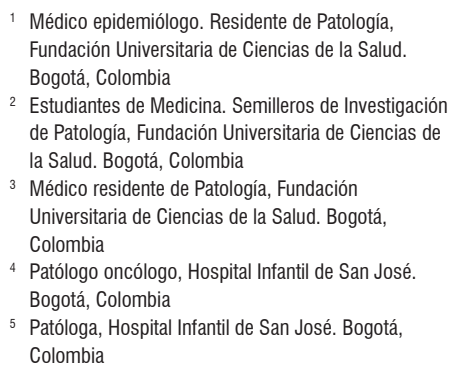

2 Estudiantes de Medicina. Semilleros de Investigación de Patología, Fundación Universitaria de Ciencias de la Salud. Bogotá, Colombia

3 Médico residente de Patología, Fundación Universitaria de Ciencias de la Salud. Bogotá, Colombia

4 Patólogo oncólogo, Hospital Infantil de San José. Bogotá, Colombia

5 Patóloga, Hospital Infantil de San José. Bogotá, Colombia

\begin{abstract}
Resumen
El leiomiosarcoma de duodeno es una patología poco frecuente con mal pronóstico. Los pacientes presentan un cuadro clínico poco específico y el diagnóstico se realiza a través del estudio histopatológico en donde se reconoce una lesión mesenquimal conformada por células tumorales malignas; en el estudio de inmunohistoquímica se observa positividad para actina de músculo liso, desmina, actina de músculo específico, caldesmon y calponina. Se presenta el caso de una mujer de 56 años con leiomiosarcoma primario de duodeno con metástasis al páncreas y al tronco celíaco.
\end{abstract}

\section{Palabras clave}

Leiomiosarcoma, duodeno, intestino delgado, tumores mesenquimales.

\begin{abstract}
Duodenal leiomyosarcoma is a rare disease with poor prognoses for patients. The disease does not have a clear set of signs and symptoms that allows easy diagnosis. Diagnosis is made on the basis of histopathological identification of a mesenchymal lesion composed of malignant tumor cells and immunohistochemistry positive for smooth muscle actin, desmin, muscle specific actin, calponin and caldesmon. We report a case of a 56 year old woman with primary duodenal leiomyosarcoma which had metastasized to the pancreas and the celiac artery.
\end{abstract}

Keywords

Leiomyosarcoma, duodenum, small intestine, mesenchymal tumors.

\section{INTRODUCCIÓN}

Los tumores mesenquimales gastrointestinales son un grupo de neoplasias con características histológicas similares pero con un patrón de inmunohistoquímica diferente $(1,2)$. La neoplasia mesenquimal más frecuente en el tracto gastrointestinal es el tumor estromal gastrointestinal (GIST), seguido de los tumores de músculo liso y los tumores neurales (1).

Los tumores gastrointestinales de músculo liso son poco frecuentes, surgen en la muscular de la mucosa y su prin- cipal localización es el esófago, seguido de colon y recto (3); estos tumores se dividen en benignos (leiomiomas) y malignos (leiomiosarcomas), siendo más infrecuentes estos últimos (3). Los leiomiosarcomas en el tracto gastrointestinal ocurren con mayor frecuencia en el estómago, representando del $0,1 \%$ al 3\% de todas las neoplasias gástricas (4), seguido por el intestino delgado (5); siendo la principal localización a este nivel en el yeyuno, se continua en el íleon, y con menos frecuencia en el duodeno (6).

Se presenta el caso de una mujer de 56 años con diagnóstico de leiomiosarcoma primario de duodeno, y además se 
realiza una revisión de la literatura de los casos reportados con un adecuado diagnóstico histopatológico.

\section{REPORTE DE CASO}

Mujer de 56 años con antecedentes de diabetes mellitus no insulinodependiente, hipertensión arterial e hipercolesterolemia, que consulta por cuadro clínico de 20 días de evolución caracterizado por epigastralgia asociado a deposiciones líquidas, emesis, astenia y pérdida de peso.

En la tomografía axial computarizada (TAC) de abdomen se evidencia, a nivel de la tercera porción del duodeno, engrosamiento mural concéntrico con lesión de márgenes irregulares que se extiende hacia los tejidos periduodenales de aproximadamente $94 \times 72 \times 48 \mathrm{~mm}$; además se identifican múltiples lesiones hipodensas de morfología redonda y ovalada en topografía del tronco celíaco (figura 1). Para complementar estudio imagenológico, se hace esofagogastroduodenoscopia y ecoen- doscopia que reportan estenosis sobre la tercera porción duodenal.

Se realiza biopsia excicional de la masa duodenal observándose en el estudio histopatológico un tumor maligno mesenquimal constituido por áreas epiteliodes y fusiformes (figura 2), con marcada atipia citológica, conteo mitótico de 27 en 10 campos de alto poder, y zonas de necrosis del $20 \%$. Además, se hace biopsia de páncreas en donde se determina compromiso por lesión tumoral (figura 3 ).

En el estudio de inmunohistoquímica se observó positividad predominantemente en las áreas fusocelulares para actina de músculo liso, desmina, calponina y coctel de queratinas; además de positividad en las áreas fusocelulares y epiteliodes para CD 99 y CD 138 (figura 4) y positividad difusa para BCL 2 con un índice de proliferación celular Ki67 del 60\%. Presentó negatividad para HMB 45, S 100, CD 117, DOG 1, CD 34, EMA, INI 1 y TL 1.

La paciente continúa hospitalizada en manejo de sus comorbilidades, con evolución tórpida y a los 20 días fallece.

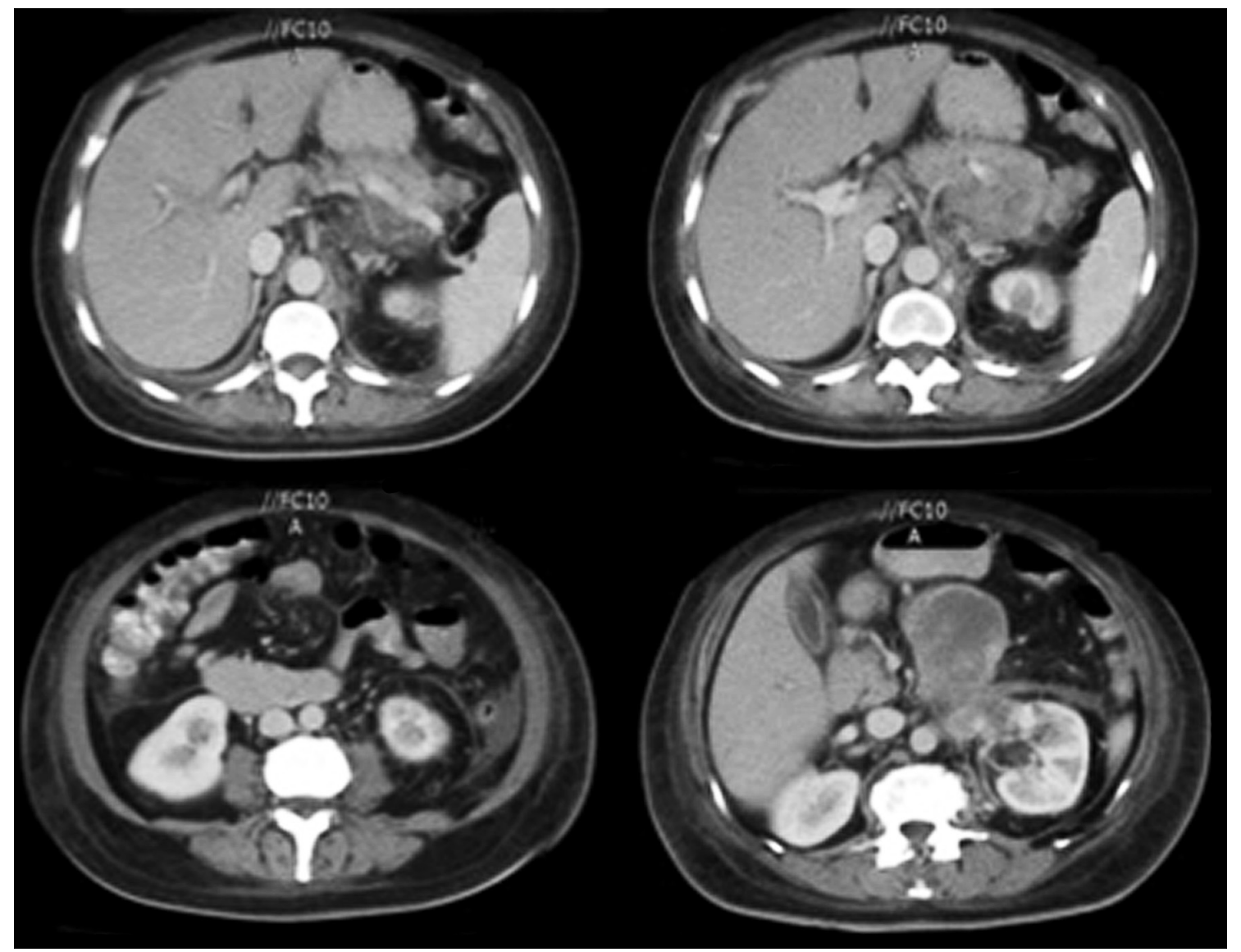

Figura 1. Masa lobulada con densidad mixta, la cual capta el medio de contraste de forma no homogénea. Produce desplazamiento de estructuras vasculares y estrechez severa por compresión extrínseca de la vena esplénica. Se observa alteración de la grasa alrededor de la masa con adenomegalias peritoneales. La masa mide aproximadamente 94 × 72 x $48 \mathrm{~mm}$. Hay líquido libre en el espacio pararrenal anterior. 

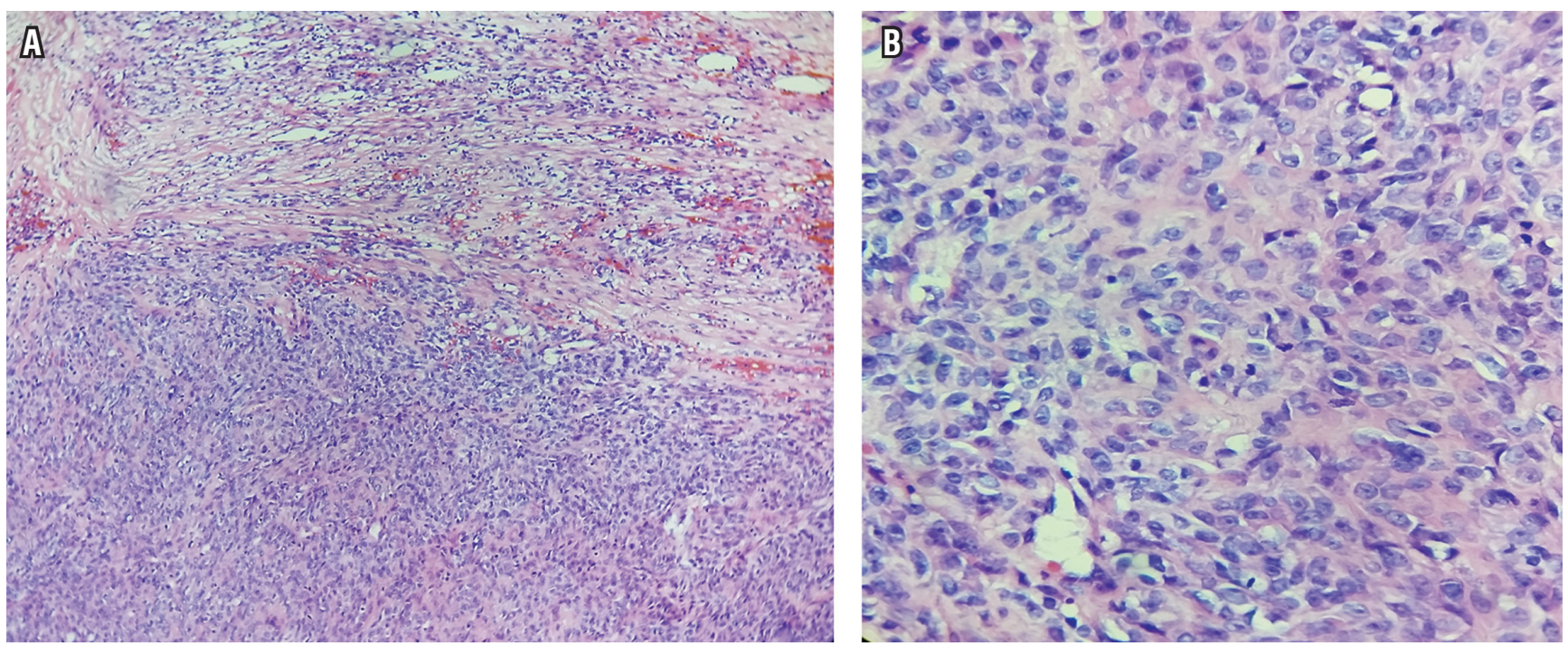

Figura 2. Fotografía de la pared duodenal. A. Pared duodenal infiltrada por lesión tumoral maligna, formada por células fusocelulares con áreas epitelioides. B. A mayor aumento se observa una marcada atipia celular con frecuentes mitosis.

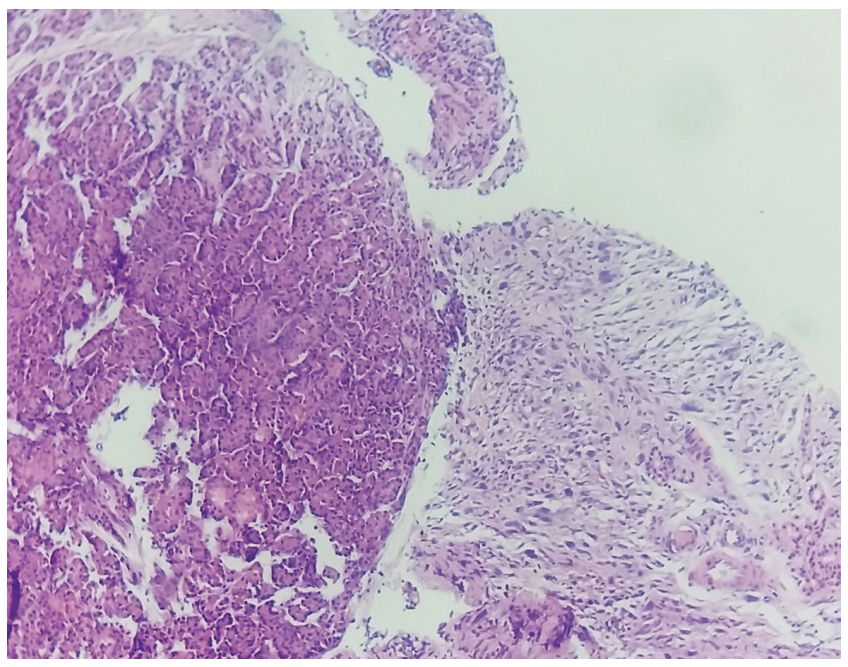

Figura 3. Parénquima pancreático comprometido por lesión tumoral maligna.

\section{DISCUSIÓN}

Los tumores mesenquimales gastrointestinales son un grupo de neoplasias que se originan de los elementos estromales de la pared del tracto gastrointestinal, con similitud histológica pero con diferencias en su perfil de inmunohistoquímica (1). Estos tumores a nivel gastrointestinal pueden clasificarse como GIST, tumores de músculo liso, tumor glómico, schwannoma, tumor miofibroblástico inflamatorio, fibromixoma plexiforme, sarcoma sinovial, sarcoma de Kaposi, entre otros (7); y se caracterizan por tener una región anatómica específica, sin ser excluyentes (8).
El primer caso reportado de leiomiosarcoma primario de duodeno fue publicado en 1920 por Von Salis (9), y en la literatura médica se han descrito aproximadamente 170 casos (6, 10-13); también se han reportado casos de leiomiosarcomas metastásicos al duodeno desde el cuerpo uterino $(14,15)$.

La edad de presentación más frecuente del leiomiosarcoma de duodeno es en la sexta década de la vida, con ligero predominio en hombres, y su principal localización es en la segunda porción del duodeno (16). Al igual que otros sarcomas, estos tumores también presentan diseminación hematógena especialmente hacia el pulmón y el hígado; también se ha descrito diseminación linfática y por continuidad comprometiendo el páncreas, el mesocolon y el colon transverso (17). Asimismo, se han reportado metástasis cutáneas y hacia el sistema musculoesquelético $(6,18)$; los pacientes con esta patología tienen mal pronóstico, con una esperanza de vida del $5 \%$ en 5 años y de $0 \%$ en 10 años $(19,20)$. Se reportó un caso de recurrencia a los 6 años que fue reintervenido con 2 años libre de enfermedad (10).

El diagnóstico clínico es complejo por su sintomatología poco específica como dolor abdominal, hemorragia de vías digestivas, obstrucción intestinal, pérdida de peso, ictericia, episodios eméticos, fiebre, diarrea, anemia, masa palpable, $y$ tractos fistulosos $(10,16)$. Las diferentes técnicas endoscópicas no siempre son conclusivas (5), y aunque exámenes como tomografías o resonancias magnéticas pueden evidenciar la masa en duodeno, el diagnóstico definitivo se realiza a través del estudio histopatológico (2), y más específicamente por inmunohistoquímica.

Macroscópicamente los leiomiosarcomas se caracterizan por presentar apariencia irregular y lobulada con sectores 

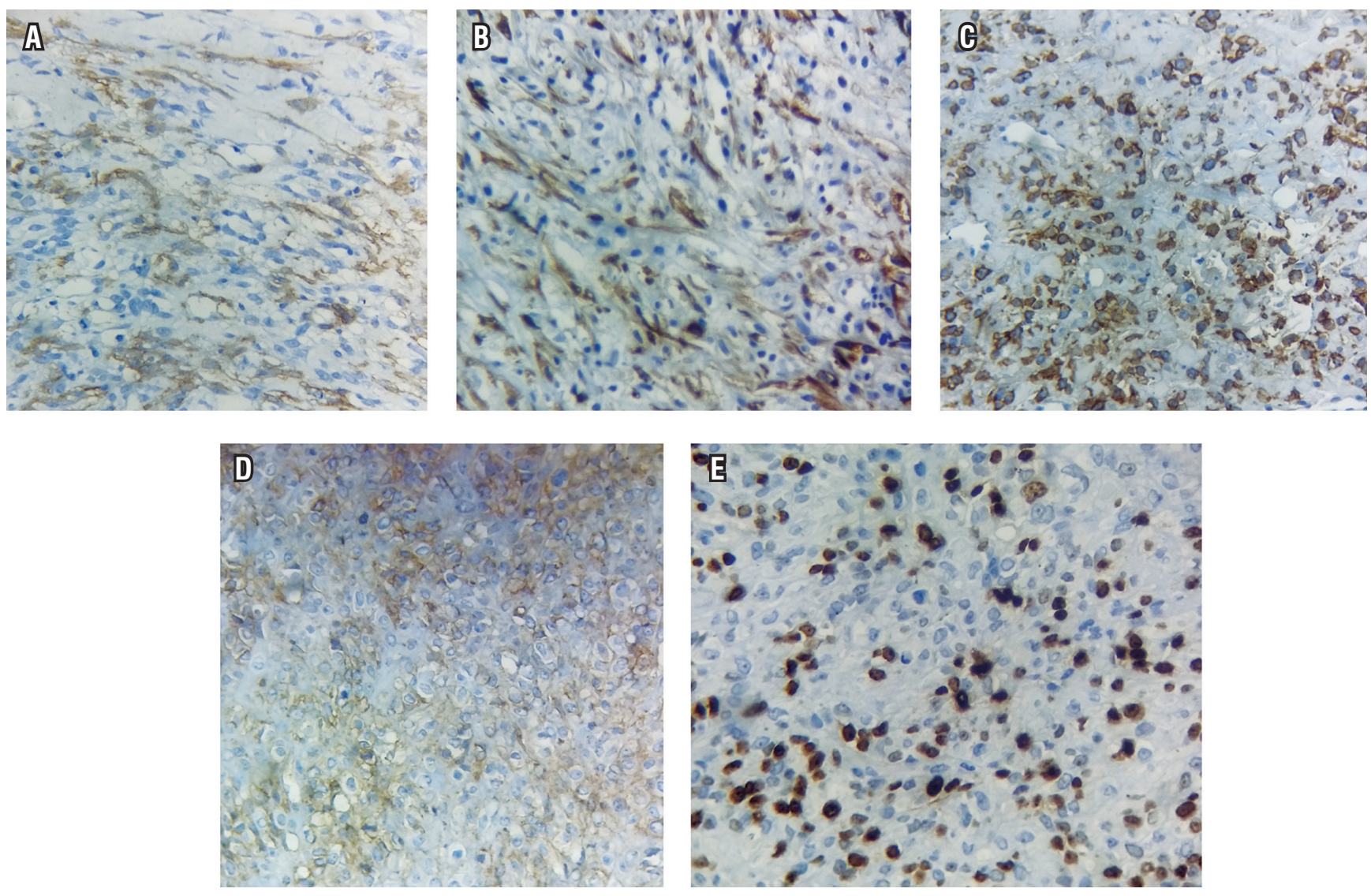

Figura 4. Estudios de inmunohistoquímica observando positividad en las células tumorales. A. Actina de músculo liso. B. Desmina. C. CD 99; D. CD 138. E. Índice de proliferación celular Ki 67 del $60 \%$.

de necrosis (21); a nivel microscópico, estos tumores exhiben un fenotipo donde claramente se distinguen de sus análogos benignos por su alta celularidad y sus características citológicas (3); el espectro morfológico puede mostrar variantes epiteliodes o de patrón fusiforme (1). Las células tumorales se caracterizan por tener un citoplasma eosinófilo con núcleos elongados e hipercromáticos con pleomorfismo nuclear (19); la actividad mitótica con frecuencia es mayor a 50 mitosis por 10 campos de alto poder; y el conteo mitótico mayor a 10 en 10 campos de alto poder asociado con el tamaño tumoral, el compromiso de planos profundos y la presencia de metástasis son indicadores de mal pronóstico $(19,20)$.

En el estudio de inmunohistoquímica, estos tumores leiomiogénicos tienen positividad para actina de músculo liso, desmina, actina de músculo específico, caldesmon, calponina y CD 34. En algunos casos se puede observar positividad para CD 138 (50\%-60\%), calretinina (25\%) y CD 99 (30\%), tanto en las áreas epiteliodes como en las fusocelulares; es infrecuente encontrar positividad para CD $117 \mathrm{y}$ HMB 45, sin embargo se pueden observar en áreas epitelio- des $(22,23)$. En algunos casos también se puede observar positividad para citoqueratinas (23).

$\mathrm{Al}$ tener en cuenta que el tumor mesenquimal más frecuente en el tracto gastrointestinal es el GIST, este debe ser el principal diagnóstico diferencial; por lo tanto, es importante el uso de la inmunohistoquímica. Más del 95\% de estos tumores expresan positividad para CD 117 y DOG 1 (24).

Whipple, en 1935, describió el tratamiento para las neoplasias de duodeno a través de una pancreatoduodenectomía (6); sin embargo, en la actualidad no existe un consenso acerca del tratamiento quirúrgico adecuado para este tipo de neoplasias. Las opciones de tratamiento son: escisión del tumor, resección segmentaria del duodeno o la pancreatoduodenectomía. El manejo con radioterapia ha demostrado tener utilidad terapéutica a nivel local, sin embargo, no se ha comprobado que tenga impacto en la sobrevida a largo plazo $(17,19)$.

\section{REFERENCIAS}

1. Miettinen M, Lasota J. Gastrointestinal stromal tumors - definition, clinical, histological, immunohistochemical, 
and molecular genetic features and differential diagnosis. Virchows Arch. 2001;438(1):1-12.

2. Yamamoto H, Handa M, Tobo T, Setsu N, Fujita K, Oshiro $\mathrm{Y}$, et al. Clinicopathological features of primary leiomyosarcoma of the gastrointestinal tract following recognition of gastrointestinal stromal tumours. Histopathology. 2013;63(2):194-207.

3. Miettinen M, Sarlomo-Rikala M, Sobin LH, Lasota J. Esophageal stromal tumors: A clinicopathologic, immunohistochemical, and molecular genetic study of 17 cases and comparison with esophageal leiomyomas and leiomyosarcomas. Am J Surg Pathol. 2000;24(2):211-22.

4. Weledji EP, Enoworock G, Ngowe MN. Gastric leiomyosarcoma as a rare cause of gastric outlet obstruction and perforation: A case report. BMC Res Notes. 2014;7:479.

5. Jabr FI, Skeik N. A leiomyosarcoma of the small bowels causing obscure gastrointestinal bleeding diagnosed by capsule endoscopy. J Med Liban. 2010;58(4):238-40.

6. Cappellani A, Di Vita M, Lo Menzo E, Zanghì A, Lanzafame $S$, Veroux P, et al. Muscular metastasis from mesocolic and duodenal leiomyosarcoma. A case report and a review of the literature. Ann Ital Chir. 2011;82(5):383-7.

7. Bosman FT, World Health O, International Agency for Research on C. WHO classification of tumours of the digestive system. Lyon: International Agency for Research on Cancer; 2010.

8. Voltaggio L, Montgomery EA. Gastrointestinal tract spindle cell lesions-just like real estate, it's all about location. Mod Pathol. 2015;28(Suppl 1):S47-66.

9. von Salis HW. Uber das Sarkom des Duodenum insbesondere das Myosarkom. Deut Z Chirurgie. 1920;160(34):180-204.

10. Colović R, Micev M, Zogović S. Leiomyosarcoma of the duodenum. A successful reoperation after recurrency. Acta Chir Iugosl. 2001;48(2):45-8.

11. Petralia GA, Hansen PD, Bowyer RC, Williamson RC. Duodenal leiomyosarcoma. Dig Surg. 1999;16(1):22-5.

12. Miettinen M, Kopczynski J, Makhlouf HR, Sarlomo-Rikala M, Gyorffy H, Burke A, et al. Gastrointestinal stromal tumors, intramural leiomyomas, and leiomyosarcomas in the duodenum: A clinicopathologic, immunohistochemical, and molecular genetic study of 167 cases. Am J Surg Pathol. $2003 ; 27(5): 625-41$.

13. Kefalas CH, Altrabulsi B, Milvenan JS, Goldschmiedt M. Endoscopic electrosurgical snare resection of leiomyosarcoma of main duodenal papilla. Gastrointest Endosc. 2004;59(6):743-5.

14. Patel JK, Cervellione KL, Sulh M, Patel AA, Gintautas J. A rare case of uterine leiomyosarcoma metastasis to the duodenum. Proc West Pharmacol Soc. 2009;52:8-10.

15. M'sakni I, Bouraoui S, Ben slama S, Lahmar-boufaroua A, Goutallier ben fadhel C, Ben sassi L, et al. Duodenal metastatic leiomyosarcoma of the uterus. A case report. Ann Chir. 2005;130(9):584-6.

16. Olurin EO, Solanke TF. Case of leiomyosarcoma of the duodenum and a review of the literature. Gut. 1968;9(6):672-7.

17. Jerraya H, Guirat A, Frikha F, Beyrouti I. Leiomyosarcoma of the duodeno-jejunal angle: Two case reports and literature review. SS Surgical Science. 2013;4(7):313-6.

18. Corcoran S, Hogan AM, Nemeth T, Bennani F, Sullivan FJ, Khan W, et al. Isolated cutaneous metastasis of uterine leiomyosarcoma: Case report and review of literature. Diagn Pathol. 2012;7:85.

19. Serrano C, George S. Leiomyosarcoma. Hematol Oncol Clin North Am. 2013;27(5):957-74.

20. Aggarwal G, Sharma S, Zheng M, Reid MD, Crosby JH, Chamberlain SM, et al. Primary leiomyosarcomas of the gastrointestinal tract in the post-gastrointestinal stromal tumor era. Ann Diagn Pathol. 2012;16(6):532-40.

21. Shenoy S. Primary small-bowel malignancy: update in tumor biology, markers, and management strategies. J Gastrointest Cancer. 2014;45(4):421-30.

22. Fenoglio-Preiser CM. Gastrointestinal pathology: An atlas and text. Philadelphia: Wolters Kluwer/Lippincott Williams \& Wilkins; 2008.

23. Dabbs DJ. Diagnostic Immunohistochemistry. 4th edition. London: Elsevier Health Sciences; 2013.

24. Corless CL. Gastrointestinal stromal tumors: What do we know now? Mod Pathol. 2014;27(Suppl 1):S1-16. 